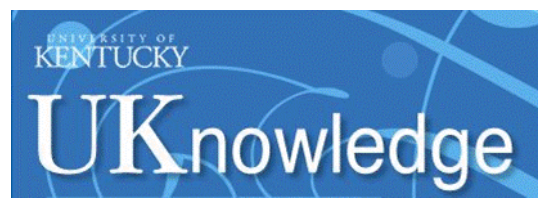

University of Kentucky

UKnowledge

$11-7-2017$

\title{
Low Arousal Positive Emotional Stimuli Attenuate Aberrant Working Memory Processing in Persons with Mild Cognitive Impairment
}

\author{
Lucas S. Broster \\ University of Kentucky, lucas.broster@uky.edu \\ Shonna L. Jenkins \\ University of Kentucky \\ Sarah D. Holmes \\ University of Kentucky \\ Gregory A. Jicha \\ University of Kentucky, gregory.jicha@uky.edu \\ Yang Jiang \\ University of Kentucky, yjiang@uky.edu \\ Follow this and additional works at: https://uknowledge.uky.edu/behavsci_facpub \\ Part of the Behavior and Behavior Mechanisms Commons, Diseases Commons, and the Neuroscience \\ and Neurobiology Commons \\ Right click to open a feedback form in a new tab to let us know how this document benefits you.
}

\section{Repository Citation}

Broster, Lucas S.; Jenkins, Shonna L.; Holmes, Sarah D.; Jicha, Gregory A.; and Jiang, Yang, "Low Arousal Positive Emotional Stimuli Attenuate Aberrant Working Memory Processing in Persons with Mild Cognitive Impairment" (2017). Behavioral Science Faculty Publications. 47.

https://uknowledge.uky.edu/behavsci_facpub/47

This Article is brought to you for free and open access by the Behavioral Science at UKnowledge. It has been accepted for inclusion in Behavioral Science Faculty Publications by an authorized administrator of UKnowledge. For more information, please contact UKnowledge@lsv.uky.edu. 


\section{Low Arousal Positive Emotional Stimuli Attenuate Aberrant Working Memory Processing in Persons with Mild Cognitive Impairment}

\section{Digital Object Identifier (DOI)}

https://doi.org/10.3233/JAD-170233

\section{Notes/Citation Information}

Published in Journal of Alzheimer's Disease, v. 60, no. 4, p. 1333-1349.

(C) 2017 - IOS Press and the authors. All rights reserved

The copyright holders have granted the permission for posting the article here.

The document available for download is the authors' post-peer-review final draft of the article.

The final publication is available at IOS Press through https://doi.org/10.3233/JAD-170233. 


\title{
Low Arousal Positive Emotional Stimuli Attenuate Aberrant Working Memory Processing in Persons with Mild Cognitive Impairment
}

\author{
Lucas S Broster ${ }^{a}$, Shonna L Jenkins ${ }^{a, b}$, Sarah D Holmes ${ }^{a, c}$, Gregory A. Jicha $^{a, d, e}$, and Yang \\ Jianga,e \\ aDepartment of Behavioral Science, University of Kentucky bMovement Disorders Program, \\ Medical University of South Carolina 'Department of Gerontology, University of Maryland \\ dDepartment of Neurology, University of Kentucky eSanders-Brown Center on Aging, University of \\ Kentucky
}

\section{Abstract}

Emotional enhancement effects on memory have been reported to mitigate the pathophysiology of Alzheimer's disease (AD). However, relative to their manifestation in persons without pathologic aging, these effects may be reduced in magnitude or even deleterious, especially in tasks that more closely model ecologic memory performance. Based upon a synthesis of such reports, we hypothesized that in persons with $\mathrm{AD}$ low arousal positive stimuli would evoke relatively intact emotional enhancement effects, but that high arousal negative stimuli would evoke disordered emotional enhancement effects. To assess this, participants with and without mild cognitive impairment (MCI) presumed to be due to $\mathrm{AD}$ performed an emotionally-valenced short-term memory task while encephalography was recorded. Results indicated that for persons with MCI, high arousal negative stimuli led to working memory processing patterns previously associated with MCI presumed due to AD and dementia of the Alzheimer's type. In contrast, low arousal positive stimuli evoked a processing pattern similar to MCI participants' unaffected spouses. Our current findings suggest that low arousal positive stimuli attenuate working memory deficits of MCI due to Alzheimer's disease.

\section{Keywords}

event-related potentials; mild cognitive impairment; Alzheimer's disease; emotional enhancement effects; working memory; affective cognition

\section{Introduction}

Alzheimer's disease (AD) is associated with severe deficits in multiple memory capacities, including deficits in working memory; however, emotional enhancement effects, the ability

Lucas Broster, MD/PhD, Aging Brain and Cognition Laboratory, Department of Behavioral Science, 116 Medical Behavioral Science Building, University of Kentucky, Lexington, KY 40536-0086, 859-338-0095, lukebroster@ gmail.com. 
for arousing, pleasant, or unsettling memories to improve encoding or subsequent retrieval of memories, appear spared in AD relative to other forms of dementia [1-7]

Despite the relative robustness of emotional enhancement effects in persons with AD, the emotional realm does change in AD. Anterior and medial temporal structures such as the amygdalae subserve emotional processing, and they experience pathophysiological change early in the course of $\mathrm{AD}[8,9]$. Further, disordered function of such limbic structures has been validated in functional imaging studies, which generally report lower-magnitude signals following emotional stimuli for individuals of advanced age or who are experiencing a stage of $\mathrm{AD}[10-15]$.

Simultaneous with neuroanatomical and functional evidence showing these AD-related deficits, emotional effects appear to enjoy maintained influence on working memory processing in the process of cognitive aging [16-20]. In particular, while certain emotional enhancement effects are attenuated over the course of aging, the influence of emotional effects on working memory in particular appear to be relatively spared [21]. In other words, emotional enhancement effects appear preserved in the context of working memory relative to emotional enhancement effects in general.

Findings of preserved emotional working memory with cognitive aging have not been universal [20]. One theory of emotional enhancement effects in AD that accounts for these discrepancies holds that in aging and dementia, emotional enhancement effects retain their normative benefits to the extent that they do not co-occur with functions that subserve executive functions such as working memory and attention [20, 22]. For example, Borg and colleagues (2011) found that relative to young adult control participants, both older individuals without dementia and older adults with dementia similarly benefited from emotional enhancement effects imparted by negatively-valenced stimuli in a simple visual recognition task, but that older adult and dementia groups showed no benefit or impairment, respectively, when negatively-valenced stimuli appeared in a task that superimposed a visuospatial binding task on the visual recognition task. The authors theorized that increased competition for domain-general neural resources led emotional enhancement effects to be replaced by emotional decrement effects [22]. This theory provides a framework for understanding a unique relationship between capacities such as working memory and emotional enhancement effects with respect to the clinical course of $\mathrm{AD}$ [20, 23].

The positivity effect of aging describes the phenomenon where positively-valenced phenomena enjoy an increasingly privileged share of attention in the context of cognitive aging, an effect most dramatic in the context of passive viewing [24-27]. Relative to younger adults, older adults attend to and remember positive stimuli better than negative stimuli, and this phenomenon has been correlated to individual differences in amygdala functional connectivity [27-29]. This finding has enjoyed an extraordinary dialectic over the previous 20 years, and multiple theories have contextualized the basis of this finding broadly including changes in motivation or neural degradation associated with cognitive aging [24, 30-33]. Literature includes reports of this phenomenon being robust to cognitive aging and $\mathrm{AD}$-spectrum disease, but also includes examples where more progression of $\mathrm{AD}$-spectrum disease led to an attenuation of this effect [33-36]. Together with the previously-discussed 
theory of resource competition, we suggest that individuals without advanced AD might preferentially process positively-valenced stimuli and consequently encounter resourcerelated difficulty when confronted with negatively-valenced stimuli. This theory is consistent with reports that aging and $\mathrm{AD}$-spectrum disease are associated with maintained benefit from stimuli of positive hedonic valence, but disordered processing of stimuli of negative hedonic valence [37-43]. It may also help contextualize the mechanism for the welldemonstrated utility of implicit cognitive interventions such as errorless learning, a cognitive intervention strategy in persons with early AD that limits the stressful emotional consequences of negative feedback to maximize the benefit of the intervention [44-47].

When assessing on-line forms of cognition such as working memory, electrophysiological methods including event-related potentials (ERP/EEG) can provide information directly based on neural activity to clarify whether differences in cognitive processing occur even in the absence of differences in behavioral output [48]. Previous work has identified a reversal in processing differences between matching and nonmatching stimuli in the P300 to P600 range of ERPs evoked by the delayed-match-to-sample task as a hallmark of AD-spectrum disease [49-51]. Further, while limited somewhat by the great variety of interpretations of what constitutes "emotional" stimuli, ERP/EEG research in the previous decades has characterized how ERPs are normatively modulated by emotionally valenced or arousing stimuli [52-57].

In the current protocol, participants with and without MCI performed a delayed-match-tosample task with emotionally-valenced stimuli to test the status of emotional enhancement effects in the context of working memory. We hypothesized that individuals with MCI would show an AD-like working memory processing effect in the electrophysiological data, and that this effect would be exacerbated in stimuli at higher levels of emotional enhancement.

\section{Methods}

\section{Participants}

32 older adult participants - 16 with amnestic mild cognitive impairment (MCI), 16 with normal cognitive status (NC) - participated in experimental protocols. All NC participants were the spouse or long-term partner of an individual in the MCI group; hence, individual MCI participants were matched with their unaffected spouse for purposes of analysis. Of the participants, 22 were members of the University of Kentucky Alzheimer's Disease Center (UK-ADC) longitudinal clinical cohort while 10 were recruited from the Kentucky Neuroscience Institute (KNI) at the University of Kentucky. Personnel involved in participant evaluation and recruitment were identical between these groups except for differences in support staff. Recruiting directly from tertiary care memory clinics reduces the risk that cognitive effects observed result from non-AD memory impairment conditions such as thyroid or vitamin $\mathrm{B}_{12}$ deficiency $[58,59]$. Individuals in the UK-ADC cohort are assessed every year (prior to clinical change) or every 6 months (subsequent to clinical change) by an interdisciplinary clinical team including a cognitive neurologist, neuropsychologist, and social worker for assessment, and they receive a battery of neuropsychological tests including the Uniform Data Set (UDS) and Geriatric Depression Scale, Short Form (GDS15). For participants who were part of the UK-ADC cohort, the 
UDS scores collected most proximal in time to research participation were consulted as descriptors of the cognitive status of participants; for participants who were recruited directly from KNI, research personnel trained in the administration of the UDS collected the UDS and GDS15 data on-site. One spousal dyad elected not to perform the on-site neuropsychological testing, so those two participants have been omitted from related analyses in this manuscript. Because other missing data were sparse, other missing neuropsychological data was handled using the expectation-maximization (EM) algorithm. Summarized neuropsychological findings and associations are included as part of Table 1.

In keeping with contemporary clinical criteria [60-63], MCI was indicated by A) absence of dementia, B) absence of cognitive, clinical, or behavioral symptoms consistent with sources of non-amnestic cognitive impairment, and C) objective memory impairment evidenced by performance more than 1.5 standard deviations below age-standardized normal values on at least one of several memory measures including Wechsler Memory Scale Logical Memory (WMS-R), the California Verbal Learning Test (CVLT-II), and the Benton Visual Retention Test (BVRT-5, Forms C \& D). AD was diagnosed using Alzheimer's Disease Dementia Workgroup criteria, which hold that insidious-onset dementia is present in the absence of another psychiatric or neurological condition [64]. All participants were recruited directly from the tertiary care setting and had received comprehensive work-up to rule-out other psychiatric or neurological causes of cognitive impairment. Individuals with MCI had been diagnosed within 12 months of data collection, all research participants had been evaluated clinically within 12 months of data collection, and all research participants were evaluated clinically on an annual basis to check for conversion to MCI. In other words, all participants were clinically evaluated both prior to and subsequent to research participation to confirm their clinical status. All participants were between age 65 and 92 with visual acuity better than 20/50 with corrective lenses in at least one eye. Exclusion criteria included history of stroke; epilepsy; head trauma; CNS infection, chronic infectious disease; psychiatric illness including substance abuse, major depression, or other mood disorder; or other neurological disease [65]. Participants taking medications known to affect cognitive function, such as sedatives or opiates, were similarly excluded.

During initial screening for recruitment, individuals who reported themselves to be lefthanded were excluded to reduce the risk that associated hemispheric ERP effects might be interpreted. However, during subsequent on-site re-screening, it was determined that two participants were born left-hand dominant, but had been forced to learn to write right-handed early in life. Because these individuals were balanced in terms of their cognitive status (i.e., one $\mathrm{NC}$, one $\mathrm{MCI}$ ), we decided not to exclude their data from the analyses.

\section{Measures and Procedures}

All participants performed an affective working memory task while ERP/EEG was recorded (Figure 1). Additionally, all but two participants either made the neuropsychological data from their most proximal UK-ADC visits available to research personnel or agreed to undergo equivalent neuropsychological testing on-site.

During each trial of the working memory task, participants were first shown two sample images surrounded by a green border and were subsequently directed to indicate whether 
sequentially-presented images matched a sample image. For each 10-12s trial, the sample image and each tested image were either uniformly low arousal positive (LAP) or high arousal negative (HAN) to prevent within-trial emotional level from being correlated to stimulus working memory status. Participants pressed the "A" and "L" keys on a keyboard to indicate matching or non-matching responses. To reduce the difficulty of the task for participants less familiar with use of keyboards, all other keys on the keyboard had been removed. Incorporating multiple memory faculties into a single paradigm, as in the emotional enhancement effect-repetition paradigm used in the current study, facilitates the interpretation of any interaction effects observed [66-68].

32 trials were performed altogether in 4 blocks of 8 trials each. Each trial included 12 stimulus presentations that required a participant response, for 384 such stimulus presentations total. Each block lasted approximately 5.5 minutes and included "rest" periods between each trial of approximately 10s. Each trial began with presentation of two sample images and was followed by tests of exactly 4 images, each tested 2-4 times, and 12 times total per trial. Images were presented in a pseudorandom sequence. The hand used to indicate a "match" response was balanced within-participants, within-dyads, and betweendyads. That is, the hand that participants would use to indicate a match was switched between blocks (e.g., if it was "A" during block 1, it would become "L" during block 2); for each dyad of participants, the initial key used to indicate a match was counterbalanced (e.g., if the participant with MCI used "A" during block 1, his or her spouse sued "L" during block 1); and for each alternating dyad of participants, the initial key used by the MCI participant to indicate a match in the dyad was counterbalanced (e.g., if the participant with MCI in the first dyad used "A" during block 1, the participant with MCI in the second dyad used "L" during block 1). Participants took a short, self-paced break between blocks that typically lasted about 60 seconds. During breaks researchers confirmed the comfort of participants and provided encouragement to participants that included reassurance about performance. Because of previous experience suggesting that negative accuracy feedback was disruptive to individuals with MCI's subsequent performance, participants received neither real-time accuracy feedback nor detailed post-hoc accuracy feedback [50].

A 5-minute practice period preceded the entire experiment to ensure that participants were comfortable with the cognitive and motor components of the task. This practice period was also designed to reduce the influence of motor learning confounds on any repetition effects. During the practice period research personnel remained in the experimental chamber with the participant and provided oral feedback related to performance. As in the subsequent 4 blocks of formal experimentation, computerized feedback was not provided.

For all but one dyad, both the participant with MCI and the unaffected spouse or partner came to the laboratory and participated on the same day. In such events, the participant with MCI participated in research protocols first, and the unaffected participant participated subsequently. While the spouse was participating in the task protocol, the participant was rescreened for eligibility and known confounds, and the UDS battery was administered if applicable. One dyad preferred to come to the laboratory separately due to scheduling conflicts, and they were the only exception to this aspect of the protocol. 


\section{Visual Stimuli}

Stimuli were 120 re-sized two-dimensional $8.3 \mathrm{~cm}$ x $5.8 \mathrm{~cm}$ IAPS images. All stimuli were presented on a high-resolution color monitor using E-prime software. Sample images were presented with a thick green outline for $3 \mathrm{~s}$, and each test stimulus was presented for $1.5 \mathrm{~s}$. Both individual images and individual trials were separated by a 1.1-1.4s jitter interval, which was employed to prevent bias in reaction time (RT) measures due to participants anticipating stimulus onset. Stimuli were presented at a $65 \mathrm{~cm}$ visual distance at a visual angle of approximately $7^{\circ}$.

IAPS images have been extensively tested and validated for numerous features including hedonic valence and arousal ratings in younger adults $[69,70]$. However, the validation of IAPS images in older adults and adults with cognitive impairment is relatively limited [71]. In particular, in older adults the hedonic valence and arousal dimensions of stimuli, which are largely independent in younger adults, become coupled such that high arousal is correlated with negative hedonic valence and low arousal is correlated with positive hedonic valence [71-73]. To account for this association, we used a multiple polynomial regression imputation algorithm to estimate the hedonic valence and arousal older adults associated with each IAPS image based on the relation between younger adults' known arousal and valence IAPS ratings and unpublished IAPS hedonic valence and arousal data scored by older adults and generously provided by Andreas Keil and colleagues. Hereafter, these scores received from Keil and colleagues are referred to as "empirical" scores. Image rating had been conducted according to the standards associated with the stimulus resource with between 27 and 56 raters per discrete image-rating session [74].

Consistent with previous reports, empirical arousal and hedonic valence values were nonindependent in older adults (Figure 2). Hence, we interpolated new adjusted arousal and hedonic valence scores for all IAPS images using a regression imputation algorithm based on the prediction model described above to establish relatively appropriate arousal and hedonic valence scores for the entire set of IAPS images. Younger adults' ratings of images' arousal and hedonic valence, the interaction between the two dimensions, and quadratic or cubic trends in the ratings of arousal and hedonic valence were used to predict older adults' empiric arousal and hedonic valence ratings, and the resultant regression equations ${ }^{1}$ were used to predict arousal and hedonic valence ratings of all IAPS images.

\footnotetext{
${ }^{1}$ The resultant equations used to impute estimates of older adults' arousal and hedonic valence of all IAPS images are listed below. All images reflect younger adult judgments of a given IAPS image unless otherwise indicated:

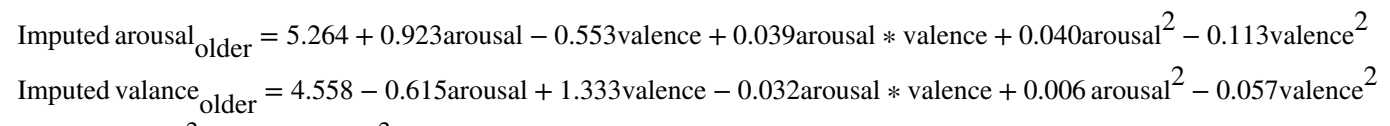

The equations were derived as described prosaically using functions similar to the form below (i.e., separately but with identical predictors for arousal and valence). Only derived $\mathrm{B}$ values associated with $p$ values $<0.1$ were included, unless its higher-order effect was significant; in this study, all excluded terms were associated with $p>0.2$ 
Based on this finding and a desire for a parsimonious design, IAPS image content was interpreted in a unidimensional fashion to ensure validity with our older adult cohort. Initially, we had planned for 3 levels along this single dimension - low arousal positive (LAP), high arousal negative (HAN), and neutral. To identify images belonging to each group, we collected images that scored within 2 points of the most extreme relevant values for LAP or HAN (i.e., images scored 1-3 or 7-9, as applicable) or that scored within 2 points of the middle value on both dimensions for neutral images (i.e., images scoring 4-6). However, upon examination of the exemplars of each class, we determined subjectively that the neutral stimulus set showed poor face validity in terms of the types of content it encompassed (e.g., including both erotica and photographs of household objects); therefore, we elected to use only 2 levels: LAP and HAN, as previously formalized.

There were a total of 1201 IAPS images at the time we planned the study. Of these, 103 and 110 images met the stated criteria for LAP or HAN, respectively, after arousal and hedonic valence value imputation (i.e., within 2 points of the most extreme value on both arousal and hedonic valence axes). 120 of the 1201 IAPS images had been rated by older adults, so about $90 \%$ of IAPS images had never been rated by older adults. Of the 120 IAPS images, 12 and 11 had met the stated criteria for LAP or HAN images, respectively."

\section{Electrophysiological Data Collection \& Preprocessing}

ERP recordings were obtained from 62 scalp sites using $\mathrm{Ag} / \mathrm{AgCl}$ electrodes embedded in an elastic cap at locations from the extended international 10-20 system. These electrodes were referenced to a midline reference electrode during recording and re-referenced to the average of the right and left mastoid potentials offline. Four additional channels were used for monitoring horizontal and vertical eye movements. Impedance was maintained below $5 \mathrm{k} \Omega$. NeuroScan hardware was used for data collection.

First, electrophysiological data were averaged according to normative protocols. Specifically, electrophysiological data were partially preprocessed using SCAN 4.5. This preprocessing consisted of manual artifact rejection, a finite impulse response filter with a band-pass of 0.05 to $40 \mathrm{~Hz}$ at $12 \mathrm{~dB} /$ octave, and epoching at -200 to $1000 \mathrm{~ms}$ relative to participant exposure to each stimulus. Epoched data were then processed further using the ERP PCA Toolkit (EP Toolkit). These steps included ocular artifact reduction using independent components analysis (ICA), motor artifact reduction, bad channel imputation, baseline-correction, and re-referencing to the average of the mastoid electrodes. These steps of analysis used all default settings of EP Toolkit, with the exception that individuals epochs were permitted to vary within $\pm 75 \mu \mathrm{V}$ of baseline rather than the default setting of $\pm 50 \mu \mathrm{V}$ of baseline. This change is normative in older adult and/or clinical populations. Such epochs were then averaged for each of the 8 experimental conditions: whether a stimulus was a

$$
\begin{aligned}
& \text { Empirical arousal }{ }_{\text {older }} / \text { valence }_{\text {older }}=\beta_{0}+\text { arousal }_{1}++ \text { valence }_{2}+\text { arousal } * \text { valence } \beta_{3}+\text { arousal }^{2} \beta_{4}+\text { valence }^{2} \beta_{5} \\
& + \text { arousal }^{3} \beta_{6}+\text { valence }^{3} \beta_{7}
\end{aligned}
$$


working memory match or non-match, whether an image was LAP or HAN, and whether an image was being tested for the first time or for an additional time.

Next, temporal principal components analysis (tPCA) was applied to the data to dissociate overlapping components present in the conventionally-averaged ERPs. In our opinion, this step was necessary in the current experiment and preferable to conventional difference waves on the grounds that individual stimuli varied on more than only psychological conditions (e.g., the LAP and HAN stimuli were not identical to one another; the images themselves differed). Promax rotation was used permit limited correlation between temporal components, following the recommendation of methodologists [75]. Retained temporal components were identified using the protocol recommended by the EP Toolkit: the averaged data were compared to a random dataset, and components that explained both greater variance than the random dataset and at least $0.5 \%$ of variance in the data were retained. Principle components reflecting classical ERP signatures were identified using topographical maps of each component, each component's temporal course, and the effect of the experimental manipulations on each component.

\section{Data Analysis}

Data were analyzed as $2 \times(2 \times 2 \times 2)$ mixed robust ANOVAs using the EP Toolkit's robust ANOVA plug-in. Effects relevant to the a priori hypothesis were first evaluated, and then post-hoc robust ANOVAs were performed for all principle components using similarlystructured robust ANOVAs. Analysis protocols recommended by the EP Toolkit documentation were used to limit the effect of multiple comparisons on these tests: each ANOVA was conducted only at an exemplar electrode of the corresponding component, and resultant $p$ values were modified by Bonferroni correction on the number of principle components tested [76]. Uncorrected $p$ value thresholds for each significant post-hoc effect are listed for each respective effect. Simple-effects models were used to interpret interaction effects. All significance values listed are based on two-tailed $p$ values except for the directional a priori hypothesis, for which one-tailed $p$ values were used. For the sake of brevity, post-hoc results failing to reach at least one-tailed significance (i.e., p > 0.1) after correction have been omitted from the report. Because participant age and education were not associated with behavioral or ERP outcomes, these variables were not retained as covariates for any models $(p s>0.2)$.

Additionally, to improve power to detect lower-order effects involving clinical group, data were analyzed as 16 dyad pairs to take advantage of shared variance attributable to similarities correlated with spousehood. Hence, behavioral data (i.e., reaction time and accuracy) and processed ERP data (i.e., as temporal principal component amplitude) were analyzed as $2 \times(2 \times 2 \times 2)$ within-dyad robust ANOVAs on cognitive status (NC or MCI), emotional enhancement effect stimulus type (LAP or HAN), working memory status (match or non-match), and repetition effect stimulus type (initial or repeated) using the EP Toolkit's robust ANOVA plug-in. Ultimately, while this analysis did decrease the $p$ value of certain effects (e.g., main effects and two-way interaction effects reliably had smaller $p$ values, but three-way interactions had larger $p$ values), the analysis was not associated with any 
categorical changes in the significance of effects in this experiment, so the analysis will not be discussed further.

\section{Results}

\section{Behavioral Results}

Mixed ANOVAs on behavioral reaction time revealed an unqualified main effect of group, $F$ $(1,28)=5.34, p=0.028, \eta_{\mathrm{p}}{ }^{2}=0.16$, such that individuals with MCI were slower to respond than were individuals without impairment (Table 2).

Mixed ANOVAs on accuracy revealed a main effect of group, $F(1,28)=16.38, p<0.001$, $\eta_{\mathrm{p}}{ }^{2}=0.37$, such that individuals with MCI were less accurate than individuals without impairment, and an Emotion $\times$ Working Memory interaction, $F(1,28)=4.41, p=0.047, \eta_{\mathrm{p}}{ }^{2}$ $=0.14$. The interaction resulted from a larger accuracy difference between working memory conditions for high arousal negative stimuli than for low arousal positive stimuli (2.5\% vs. $0.1 \%)$. Other effects were non-significant.

\section{Conventionally-Averaged Waveforms}

Conventionally-averaged ERP/EEGs were examined to ensure data integrity. These data had experienced all processing described in the Methods section other than tPCA. The conventionally-averaged waveforms indeed showed classical components including a P3, frontal N400, posterior P2, and late positive component (LPC) (Figure 3). The latency of individual components appeared somewhat faster than is typical, perhaps owing to the relatively stringent time-pressure in the task.

Because the a priori hypothesis specifically involving the experimental conditions related to working memory and emotional enhancement effects, we next examined conventional difference waves of working memory effects (Match - Nonmatch) for LAP and HAN stimuli for each clinical group to assess for differences in data quality that might be related to particular within-subject conditions (Figure 4).

\section{Temporal Principal Components Analysis}

The primary principal components associated with the experiment corresponded to classical ERP components, including the P2, P3, frontal N400, P600, and late positive potential (LPP) (e.g., as similarly reflected in Figure 3). Further, the tPCA solution suggested that LPC visible in the conventionally-averaged waveform was actually composed of discrete overlapping phenomena: one peaking near $600 \mathrm{~ms}$, and one peaking near the end of the epoched time-window. Because the late repetition effect represented discrete effects rather than a monolith, statistical evaluation of the experimental data was restricted to analysis of the individual temporal components of the tPCA solution.

\section{A Priori Analysis}

The P600 was associated with a significant Group $\times$ Emotion $\times$ Match three-way interaction, $T_{W J t} / c(1.0,23.3)=8.95, p=0.011$, resulting from an Emotion $\times$ Match interaction for individuals with MCI, $T_{W J} / c(1.0,15.0)=7.54, p=0.027$, but not for individuals without 
impairment. In individuals without impairment, matching stimuli were associated with a larger P600 than were nonmatching stimuli regardless of emotional content of stimuli, $T_{W J l} / \mathcal{C}(1.0,15.0)=18.39, p=0.0032$, but in individuals with MCI, this pattern was present for positive stimuli, $T_{W J t} / \mathcal{C}(1.0,15.0)=10.41, p=0.0079$, but it was absent for negative stimuli, $T_{W J t} / c(1.0,15.0)=1.26, p=0.28$, and the direction of this effect trended in the opposite direction for negative stimuli in this group (Figure 5B).

\section{Post-Hoc Electrophysiological Analyses}

For these analysis, Bonferroni correction was applied on the number of principle components retained (7), resulting in a $p$ value cut-off of 0.007 . Within these bounds, the frontal N400 component was associated with a main effect of emotion, $T_{W J} / c(1.0,30.0)=$ $10.17, p=0.0059$, such that the component was larger for negative stimuli, and a main effect of repetition, $T_{W J l} / c(1.0,22.3)=7.05, p=0.010$, such that the component was more negative upon repetition. Other effects were non-significant after Bonferroni correction.

\section{Integrated Analyses}

Individual neuropsychological data were not significantly correlated with behavioral working memory reaction time effects or with the electrophysiological working memory effects at the P600 after accounting for multiple comparisons (Table 1, bottom rows). Additionally, behavioral working memory effects were not significantly correlated with the corresponding electrophysiological P600 working memory effects.

\section{Discussion}

We found that persons with MCI showed AD-like ERPs when performing working memory with high arousal negative (HAN) emotional stimuli, but showed ERPs similar to persons without impairment for low arousal positive (LAP) stimuli. Persons with MCI were also slower and less accurate than persons without impairment. This was consistent with our a priori hypothesis that HAN stimuli more than LAN stimuli would lead to working memory processing reflective of the influence of $\mathrm{AD}$.

We were surprised by the degree of similarity in the working memory processing of persons with and without MCI for LAP stimuli. In a previous task similar to the current protocol that differed mainly in that it used simple line drawings without appreciate hedonic valence or arousal as visual stimuli, participants with MCI showed a P3 similar to the HAN result in the current protocol [49]. While we did predict that LAP stimuli would have smaller between group differences than HAN stimuli, we anticipated that the greater complexity of the IAPS images in the current protocol relative to simple line-drawings would tax executive resources such that persons with MCI showing a NC-like working memory processing pattern would be extremely unlikely. This finding may suggest that, rather than merely being associated with a milder degree of working memory dysregulation, LAP stimuli may normalize working memory processing in persons with MCI, even for complex stimuli. In other words, the translatability of this finding to still-more-ecologically-valid phenomena is relatively fathomable. 
Put together, these findings support the idea that stressful circumstances disrupt the normal effects of emotional enhancement on working memory, but they stop short of suggesting that emotional enhancement effects disrupt cognition in the context of MCI in general. Instead, based on the contrast of the current results with similar studies that used simpler stimuli that were non-emotional, the current results suggest that LAP environments maintain the ability to facilitate normal working memory processing in persons with MCI. By extension, LAP environments likely also maintain the ability to facilitate normal processing in aspects of cognition subserved by working memory. Future work should evaluate the extent to which LAP environments or stimuli have beneficial effects in contexts beyond working memory to confirm this possibility. The current results may support a neural basis for recent investigations of the impact of mindfulness training in persons with cognitive change due to AD [77-81]. Specifically, therapeutic effects of mindfulness may be attributable in part to the generation of a mental status relatively similar to that evoked by the low arousal positive stimuli utilized in the current experiment.

One theory that could contextualize the current results is the dual competition model, which proposes a multi-axial system of dependencies between cognition and emotion that modulate motivation and behavior [82-85]. In the current experiment, this model predicts that HAN stimuli, generally being emotional content high in threat, would tend to bias neural resource allocation toward analysis of the content of the stimuli themselves rather than toward completion of the current working memory task, with consequential effects on processing dependent upon total resource capacity. This could account for the relatively small difference in P600 between working memory conditions for HAN stimuli in persons with MCI at frontal sites. However, our findings suggest that a posterior mechanism may play a role in working memory processing in such a context.

Another theory that could contextualize the current results is arousal-biased competition, which holds that in high arousal states processing of relevant information is enhanced, but processing of distracting information is impaired [86-90]. In the current study, participants showed a larger P600 for matching stimuli than for non-matching stimuli in all circumstances except for HAN stimuli processed by persons with MCI. For that group, the pattern trended in the opposite direction. Impaired inhibition of non-matching stimuli unique to the high arousal context could account for this result. However, we did not find clear evidence of processing benefit of relevant stimuli. One possibility for this discrepancy may be that "improved" processing of a relevant (i.e., matching) HAN stimulus is paradoxically reflected in processing of stimulus characteristics rather than its working memory retrieval status.

In this study, stimuli were selected along a bimodal, unidimensional hedonic valence-arousal axis due to data suggesting that these features became more and more closely correlated over the course of aging. Consequently, we cannot differentiate effects of negative hedonic valence and high arousal in the current study. Some have suggested that despite the apparent collinearity of hedonic valence and arousal judgments by older adults, physiological effects of these dimensions remain distinct [91]. Consequently, we suggest that follow-up research might attempt to disentangle the influence of the arousal and hedonic valence dimensions of stimuli on the presence of the AD-like cognitive signature replicated in the current study 
[49]. Based on our imputation, it should be possible to select stimuli of moderate arousal that differ somewhat in hedonic valence. In our opinion, because the non-emotional stimuli used in the previous study were estimated to have neutral hedonic valence and low arousal, we suggest that the relative salutary effect of LAP stimuli in the current study might have been due mostly to positive hedonic valence. Tantalizingly parallel to this view is the abundant literature on positivity effects in aging and the related dialectic on their presence or absence in persons with AD-spectrum illness.

Emotional stimuli within each trial of this experiment were uniformly low arousal positive or high arousal negative. As a result, each trial consisted of about 10-12 seconds of consistently-valenced emotional stimuli. This persistence could be sufficient to induce a mood, which might produce psychological and electrophysiological effects distinct from that caused by the emotional stimuli themselves. In our opinion, this possibility is unlikely as it would predict an Emotion $\times$ Repetition or Emotion $\times$ Working Memory $\times$ Repetition interaction that reflected differential processing after mood induction, but such an effect was not observed in the results of this study. However, it is possible that available power limited the ability to detect such an effect.

Some studies of ERPs evoked by emotional stimuli have reported emotional effects at latencies as early as the P1, which would be an earlier latency than the latencies found in the current study [55]. However, such studies differed in design by the inclusion of neutral stimuli, and it was typically only in contrasts with non-emotional, neutral stimuli that the earliest latency emotional effects were observed, though some later latency effects were similarly constrained to contrasts between emotional and non-emotionally stimuli $[55,56]$. In this experiment, such stimuli were excluded because of perceived poor face validity among stimuli rated neutrally on both hedonic valence and arousal, and hedonic valence and arousal were not treated dimensionally because of evidence that those factors were collinear in older adults and persons with MCI. However, since other physiological correlates of the dimensional independence of hedonic valence and arousal appear intact despite correlation in the self-reported ratings of emotional images, ERPs associated with hedonic valence and arousal may likewise remain independent. Future studies should test this possibility as a way to assess the neural mechanisms of burgeoning collinearity between these factors [72]. In the current study, the working memory effects of interest manifested primarily at a later component (P600), but in the similar study that used non-emotional stimuli, the effect occurred somewhat earlier during P3 [49, 51]. Multiple differences between the two studies could account for this discrepancy. First, the use of emotional stimuli classically evokes a prominent late component called the late positive potential (LPP), which is absent or subtle in experiments without emotional effects [53]. Therefore, differences in stimulus characteristics could account for the discrepancy. Second, the current study used PCA to disentangle overlapping components, but the previous study used a conventional analysis approach [51]. The PCA approach may have identified the true source of the apparent variance in the working memory effect more accurately.

ERPs associated with working memory were not correlated with neuropsychological or behavioral outcomes in the current study. This finding replicates classical findings that correlations between particular ERP components and behavioral outcomes such as reaction 
time breaks down when coupled with cognitive tasks that engage multiple memory systems $[92,93]$.

The post-hoc finding that the N400 was associated with more negative values for HAN stimuli replicates some similar findings in the affective priming literature [94]. N400 effects are classically evoked by semantic violations in the context of language-based semantic priming paradigms, but have also been linked to other cognitive domains that researchers have analogized to language semantics, especially processes that denote violation of systematic rules, including phenomena that evoke moral disgust [95-98]. As such, the larger N400 identified in the current study may represent a complex evaluation of inexcusable moral circumstances depicted in a HAN stimulus rather than a mere categorization of the stimulus on the dimensions of arousal and hedonic valence. This possibility may help contextualize why repetition was associated with larger N400 amplitudes rather than the smaller N400 amplitudes that generally accompany repetition of N400-relevant semantic violations. Whereas repeating a semantic violation may normalize it, the repetition of circumstances that promote moral outrage may rouse increased scrutiny. Future studies could attempt to modulate the sense of moral outrage evoked by some HAN stimuli while controlling for subjective arousal and hedonic valence to assess the true determinant of N400 magnitude in such stimuli.

Participants in this experiment were screened for depressive symptoms, and individuals with current depressive symptoms were not enrolled in the study. Because the rate of depression in the general population with cognitive change due to Alzheimer's disease is high and some evidence suggests that individuals with depression or remitted depression show a visual attention bias toward negative stimuli, this may suggest that the current results may be limited in the extent to which they are externally valid to the patient population with cognitive change due to Alzheimer's disease [90, 99-101]. Future studies could investigate any moderating effects of individual depressive states on the current results.

This experiment excluded individuals taking certain categories of psychoactive drugs, but individuals with mild cognitive impairment were uniformly taking donepezil or rivastigmine as part of their regular medical regimen as treatment for the changes to their memory and thinking [102]. These medications have known effects on ERP waveforms, so a subset of group differences identified in this study could be attributable to such differences [103, 104]. Because the differences in ERPs in this experiment were associated with interactions between experimental conditions and groups, the relevance of this issue to the main findings of this manuscript is limited. However, care should be exercised in the interpretation of apparent simple group differences in the conventionally-averaged data.

We report evidence that individuals with MCI presumed due to the Alzheimer's disease show disordered working memory processing of HAN stimuli, but normal processing of LAP stimuli. We suggest that these findings are relevant to ongoing disputes in the literature regarding the status and viability of emotional enhancement effects in MCI and AD. Further, we suggest that clinical interventions that produce a low arousal positive-like environment may reduce the functional impact of the early stages of $\mathrm{AD}$. 


\section{Acknowledgments}

We would like to thank P Lang and A Keil for providing advice related to potential considerations in stimulus selection and categorization and J Dien for ongoing assistance in appropriate use of the ERP PCA Toolkit. We would like to thank F Schmitt, R Kryscio, E Abner, and the University of Kentucky Alzheimer Disease Center (UKADC) for their help with the compilation of the neuropsychological test results. We would like to thank M Mather and two anonymous reviewers for helpful recommendations and criticisms during review. This work was supported by funding from the National Institutes of Health (5 T32 AG 242-18; P30 AG028383; UL1RR033173;

UL1TR000117). We have no financial or material conflicts of interest to report.

\section{References}

1. Lavenu I, Pasquier F, Lebert F, Petit H, Van der Linden M. Perception of emotion in frontotemporal dementia and Alzheimer disease. Alzheimer Dis Assoc Disord. 1999; 13:96-101. [PubMed: 10372953]

2. Fernandez-Duque D, Black SE. Impaired recognition of negative facial emotions in patients with frontotemporal dementia. Neuropsychologia. 2005; 43:1673-1687. [PubMed: 16009249]

3. Balconi M, Cotelli M, Brambilla M, Manenti R, Cosseddu M, Premi E, Gasparotti R, Zanetti O, Padovani A, Borroni B. Understanding Emotions in Frontotemporal Dementia: The Explicit and Implicit Emotional Cue Mismatch. J Alzheimers Dis. 2015

4. Kumfor F, Irish M, Hodges JR, Piguet O. Frontal and temporal lobe contributions to emotional enhancement of memory in behavioral-variant frontotemporal dementia and Alzheimer's disease. Front Behav Neurosci. 2014; 8:225. [PubMed: 25009480]

5. Joshi A, Barsuglia JP, Mather MJ, Jimenez EE, Shapira J, Mendez MF. Evaluation of emotional blunting in behavioral variant frontotemporal dementia compared to Alzheimer's disease. Dement Geriatr Cogn Disord. 2014; 38:79-88. [PubMed: 24603498]

6. Bertoux M, de Souza LC, Sarazin M, Funkiewiez A, Dubois B, Hornberger M. How Preserved is Emotion Recognition in Alzheimer Disease Compared With Behavioral Variant Frontotemporal Dementia? Alzheimer Dis Assoc Disord. 2014

7. Miller LA, Hsieh S, Lah S, Savage S, Hodges JR, Piguet O. One size does not fit all: face emotion processing impairments in semantic dementia, behavioural-variant frontotemporal dementia and Alzheimer's disease are mediated by distinct cognitive deficits. Behav Neurol. 2012; 25:53-60. [PubMed: 22207423]

8. Braak H, Alafuzoff I, Arzberger T, Kretzschmar H, Del Tredici K. Staging of Alzheimer diseaseassociated neurofibrillary pathology using paraffin sections and immunocytochemistry. Acta Neuropathol. 2006; 112:389-404. [PubMed: 16906426]

9. Braak H, Braak E. Neuropathological stageing of Alzheimer-related changes. Acta Neuropathol. 1991; 82:239-259. [PubMed: 1759558]

10. Kensinger EA, Brierley B, Medford N, Growdon JH, Corkin S. Effects of normal aging and Alzheimer's disease on emotional memory. Emotion. 2002; 2:118-134. [PubMed: 12899186]

11. Leclerc CM, Kensinger EA. Neural processing of emotional pictures and words: a comparison of young and older adults. Dev Neuropsychol. 2011; 36:519-538. [PubMed: 21516546]

12. Kensinger, EA. Emotional Memory across the Adult Lifespan. Psychology Press; 2008.

13. Waring JD, Seiger AN, Solomon PR, Budson AE, Kensinger EA. Memory for the 2008 presidential election in healthy ageing and mild cognitive impairment. Cogn Emot. 2014; 28:1407-1421. [PubMed: 24533684]

14. Budson AE, Todman RW, Chong H, Adams EH, Kensinger EA, Krangel TS, Wright CI. False recognition of emotional word lists in aging and Alzheimer disease. Cogn Behav Neurol. 2006; 19:71-78. [PubMed: 16783129]

15. Kensinger EA, Anderson A, Growdon JH, Corkin S. Effects of Alzheimer disease on memory for verbal emotional information. Neuropsychologia. 2004; 42:791-800. [PubMed: 15037057]

16. Kensinger EA, Corkin S. Effect of negative emotional content on working memory and long-term memory. Emotion. 2003; 3:378-393. [PubMed: 14674830]

17. Waring JD, Payne JD, Schacter DL, Kensinger EA. Impact of individual differences upon emotioninduced memory trade-offs. Cogn Emot. 2010; 24:150-167. [PubMed: 20161513] 
18. Freeman D, Startup H, Dunn G, Cernis E, Wingham G, Pugh K, Cordwell J, Kingdon D. The interaction of affective with psychotic processes: a test of the effects of worrying on working memory, jumping to conclusions, and anomalies of experience in patients with persecutory delusions. J Psychiatr Res. 2013; 47:1837-1842. [PubMed: 23871449]

19. Kerestes R, Ladouceur CD, Meda S, Nathan PJ, Blumberg HP, Maloney K, Ruf B, Saricicek A, Pearlson GD, Bhagwagar Z, Phillips ML. Abnormal prefrontal activity subserving attentional control of emotion in remitted depressed patients during a working memory task with emotional distracters. Psychol Med. 2012; 42:29-40. [PubMed: 21733287]

20. Broster LS, Blonder LX, Jiang Y. Does emotional memory enhancement assist the memoryimpaired? Front Aging Neurosci. 2012; 4:2. [PubMed: 22479245]

21. Mikels JA, Larkin GR, Reuter-Lorenz PA, Cartensen LL. Divergent trajectories in the aging mind: changes in working memory for affective versus visual information with age. Psychol Aging. 2005; 20:542-553. [PubMed: 16420130]

22. Borg C, Leroy N, Favre E, Laurent B, Thomas-Anterion C. How emotional pictures influence visuospatial binding in short-term memory in ageing and Alzheimer's disease? Brain Cogn. 2011; 76:20-25. [PubMed: 21481999]

23. Knight M, Mather M. Reconciling findings of emotion-induced memory enhancement and impairment of preceding items. Emotion. 2009; 9:763-781. [PubMed: 20001121]

24. Mather M, Carstensen LL. Aging and motivated cognition: the positivity effect in attention and memory. Trends Cogn Sci. 2005; 9:496-502. [PubMed: 16154382]

25. Charles ST, Mather M, Carstensen LL. Aging and emotional memory: the forgettable nature of negative images for older adults. J Exp Psychol Gen. 2003; 132:310-324. [PubMed: 12825643]

26. Reed AE, Chan L, Mikels JA. Meta-analysis of the age-related positivity effect: age differences in preferences for positive over negative information. Psychol Aging. 2014; 29:1-15. [PubMed: 24660792]

27. Mather M, Canli T, English T, Whitfield S, Wais P, Ochsner K, Gabrieli JD, Carstensen LL. Amygdala responses to emotionally valenced stimuli in older and younger adults. Psychol Sci. 2004; 15:259-263. [PubMed: 15043644]

28. Reed AE, Carstensen LL. The theory behind the age-related positivity effect. Front Psychol. 2012; 3:339. [PubMed: 23060825]

29. Sakaki M, Nga L, Mather M. Amygdala functional connectivity with medial prefrontal cortex at rest predicts the positivity effect in older adults' memory. J Cogn Neurosci. 2013; 25:1206-1224. [PubMed: 23530897]

30. Carstensen LL, Pasupathi M, Mayr U, Nesselroade JR. Emotional experience in everyday life across the adult life span. J Pers Soc Psychol. 2000; 79:644-655. [PubMed: 11045744]

31. Nashiro K, Sakaki M, Mather M. Age differences in brain activity during emotion processing: reflections of age-related decline or increased emotion regulation? Gerontology. 2012; 58:156163. [PubMed: 21691052]

32. Carstensen LL. Motivation for social contact across the life span: a theory of socioemotional selectivity. Nebr Symp Motiv. 1992; 40:209-254. [PubMed: 1340521]

33. Bohn L, Kwong See ST, Fung HH. Time perspective and positivity effects in Alzheimer's disease. Psychol Aging. 2016; 31:574-582. [PubMed: 26974590]

34. Kalenzaga S, Lamidey V, Ergis AM, Clarys D, Piolino P. The positivity bias in aging: Motivation or degradation? Emotion. 2016; 16:602-610. [PubMed: 26950366]

35. Leal SL, Noche JA, Murray EA, Yassa MA. Positivity effect specific to older adults with subclinical memory impairment. Learn Mem. 2016; 23:415-421. [PubMed: 27421893]

36. Cuddy LL, Sikka R, Silveira K, Bai S, Vanstone A, Walla P. Music-evoked autobiographical memories (MEAMs) in Alzheimer disease: Evidence for a positivity effect. Cogent Psychology. 2017; 4:1277578.

37. Sava AA, Paquet C, Krolak-Salmon P, Dumurgier J, Hugon J, Chainay H. Emotional memory enhancement in respect of positive visual stimuli in Alzheimer's disease emerges after rich and deep encoding. Cortex. 2015; 65:89-101. [PubMed: 25681651] 
38. LaBar KS, Torpey DC, Cook CA, Johnson SR, Warren LH, Burke JR, Welsh-Bohmer KA. Emotional enhancement of perceptual priming is preserved in aging and early-stage Alzheimer's disease. Neuropsychologia. 2005; 43:1824-1837. [PubMed: 16154458]

39. Kazui H, Mori E, Hashimoto M, Hirono N. Enhancement of declarative memory by emotional arousal and visual memory function in Alzheimer's disease. J Neuropsychiatry Clin Neurosci. 2003; 15:221-226. [PubMed: 12724465]

40. Hamann S, Monarch ES, Goldstein FC. Impaired fear conditioning in Alzheimer's disease. Neuropsychologia. 2002; 40:1187-1195. [PubMed: 11931922]

41. Boller F, El Massioui F, Devouche E, Traykov L, Pomati S, Starkstein SE. Processing emotional information in Alzheimer's disease: effects on memory performance and neurophysiological correlates. Dement Geriatr Cogn Disord. 2002; 14:104-112. [PubMed: 12145458]

42. Kazui H, Mori E, Hashimoto M, Hirono N, Imamura T, Tanimukai S, Hanihara T, Cahill L. Impact of emotion on memory. Controlled study of the influence of emotionally charged material on declarative memory in Alzheimer's disease. Br J Psychiatry. 2000; 177:343-347. [PubMed: 11116776]

43. Ikeda M, Mori E, Hirono N, Imamura T, Shimomura T, Ikejiri Y, Yamashita H. Amnestic people with Alzheimer's disease who remembered the Kobe earthquake. Br J Psychiatry. 1998; 172:425428. [PubMed: 9747405]

44. White L, Ford MP, Brown CJ, Peel C, Triebel KL. Facilitating the use of implicit memory and learning in the physical therapy management of individuals with Alzheimer disease: a case series. J Geriatr Phys Ther. 2014; 37:35-44. [PubMed: 23459238]

45. Lee GY, Yip CC, Yu EC, Man DW. Evaluation of a computer-assisted errorless learning-based memory training program for patients with early Alzheimer's disease in Hong Kong: a pilot study. Clin Interv Aging. 2013; 8:623-633. [PubMed: 23766638]

46. Jean L, Simard M, Wiederkehr S, Bergeron ME, Turgeon Y, Hudon C, Tremblay I, van Reekum R. Efficacy of a cognitive training programme for mild cognitive impairment: results of a randomised controlled study. Neuropsychol Rehabil. 2010; 20:377-405. [PubMed: 20029715]

47. Kessels RP, de Haan EH. Mnemonic strategies in older people: a comparison of errorless and errorful learning. Age Ageing. 2003; 32:529-533. [PubMed: 12958003]

48. Guo C, Lawson AL, Zhang Q, Jiang Y. Brain potentials distinguish new and studied objects during working memory. Hum Brain Mapp. 2008; 29:441-452. [PubMed: 17497630]

49. Broster, LS., Li, J., Smith, C., Jicha, GA., Munro, N., Hively, L., Jiang, Y. Annual Meeting of the Society for Neuroscience. Washington, DC: 2011.

50. Broster LS, Li J, Smith CD, Jicha GA, Schmitt FA, Jiang Y. Repeated retrieval during working memory is sensitive to amnestic mild cognitive impairment. J Clin Exp Neuropsychol. 2013; 35:946-959. [PubMed: 24074205]

51. Li J, Broster LS, Jicha GA, Munro NB, Schmitt FA, Abner E, Kryscio R, Smith CD, Jiang Y. A cognitive electrophysiological signature differentiates amnestic mild cognitive impairment from normal aging. Alzheimers Res Ther. 2017; 9:3. [PubMed: 28100252]

52. Schupp HT, Stockburger J, Codispoti M, Junghofer M, Weike AI, Hamm AO. Stimulus novelty and emotion perception: the near absence of habituation in the visual cortex. Neuroreport. 2006; 17:365-369. [PubMed: 16514360]

53. Rozenkrants B, Olofsson JK, Polich J. Affective visual event-related potentials: arousal, valence, and repetition effects for normal and distorted pictures. Int J Psychophysiol. 2008; 67:114-123. [PubMed: 18160161]

54. Mendez-Bertolo C, Pozo MA, Hinojosa JA. Early effects of emotion on word immediate repetition priming: electrophysiological and source localization evidence. Cogn Affect Behav Neurosci. 2011; 11:652-665. [PubMed: 21922349]

55. Foti D, Hajcak G, Dien J. Differentiating neural responses to emotional pictures: evidence from temporal-spatial PCA. Psychophysiology. 2009; 46:521-530. [PubMed: 19496228]

56. Schupp HT, Junghofer M, Weike AI, Hamm AO. Attention and emotion: an ERP analysis of facilitated emotional stimulus processing. Neuroreport. 2003; 14:1107-1110. [PubMed: 12821791] 
57. Feng C, Li W, Tian T, Luo Y, Gu R, Zhou C, Luo YJ. Arousal modulates valence effects on both early and late stages of affective picture processing in a passive viewing task. Soc Neurosci. 2014; 9:364-377. [PubMed: 24601745]

58. Jicha GA, Abner E, Schmitt FA, Cooper GE, Stiles N, Hamon R, Carr S, Smith CD, Markesbery WR. Clinical features of mild cognitive impairment differ in the research and tertiary clinic settings. Dement Geriatr Cogn Disord. 2008; 26:187-192. [PubMed: 18724049]

59. Luck T, Riedel-Heller SG, Kaduszkiewicz H, Bickel H, Jessen F, Pentzek M, Wiese B, Koelsch H, van den Bussche H, Abholz HH, Moesch E, Gorfer S, Angermeyer MC, Maier W, Weyerer S. AgeCoDe g. Mild cognitive impairment in general practice: age-specific prevalence and correlate results from the German study on ageing, cognition and dementia in primary care patients (AgeCoDe). Dement Geriatr Cogn Disord. 2007; 24:307-316. [PubMed: 17848793]

60. Albert MS, DeKosky ST, Dickson D, Dubois B, Feldman HH, Fox NC, Gamst A, Holtzman DM, Jagust WJ, Petersen RC, Snyder PJ, Carrillo MC, Thies B, Phelps CH. The diagnosis of mild cognitive impairment due to Alzheimer's disease: recommendations from the National Institute on Aging-Alzheimer's Association workgroups on diagnostic guidelines for Alzheimer's disease. Alzheimers Dement. 2011; 7:270-279. [PubMed: 21514249]

61. Arsenault-Lapierre G, Whitehead V, Belleville S, Massoud F, Bergman H, Chertkow H. Mild cognitive impairment subcategories depend on the source of norms. J Clin Exp Neuropsychol. 2011; 33:596-603. [PubMed: 21302169]

62. Lekeu F, Magis D, Marique P, Delbeuck X, Bechet S, Guillaume B, Adam S, Petermans J, Moonen G, Salmon E. The California Verbal Learning Test and other standard clinical neuropsychological tests to predict conversion from mild memory impairment to dementia. J Clin Exp Neuropsychol. 2010; 32:164-173. [PubMed: 19459119]

63. Reid LM, Maclullich AM. Subjective memory complaints and cognitive impairment in older people. Dement Geriatr Cogn Disord. 2006; 22:471-485. [PubMed: 17047326]

64. McKhann GM, Knopman DS, Chertkow H, Hyman BT, Jack CR Jr, Kawas CH, Klunk WE, Koroshetz WJ, Manly JJ, Mayeux R, Mohs RC, Morris JC, Rossor MN, Scheltens P, Carrillo MC, Thies B, Weintraub S, Phelps CH. The diagnosis of dementia due to Alzheimer's disease: recommendations from the National Institute on Aging-Alzheimer's Association workgroups on diagnostic guidelines for Alzheimer's disease. Alzheimers Dement. 2011; 7:263-269. [PubMed: 21514250]

65. Robert PH, Berr C, Volteau M, Bertogliati C, Benoit M, Mahieux F, Legrain S, Dubois B, Pre ALS. Neuropsychological performance in mild cognitive impairment with and without apathy. Dement Geriatr Cogn Disord. 2006; 21:192-197. [PubMed: 16424666]

66. Kennedy KM, Rodrigue KM, Head D, Gunning-Dixon F, Raz N. Neuroanatomical and cognitive mediators of age-related differences in perceptual priming and learning. Neuropsychology. 2009; 23:475-491. [PubMed: 19586211]

67. Voss JL, Paller KA. An electrophysiological signature of unconscious recognition memory. Nat Neurosci. 2009; 12:349-355. [PubMed: 19198606]

68. Voss JL, Paller KA. Brain substrates of implicit and explicit memory: the importance of concurrently acquired neural signals of both memory types. Neuropsychologia. 2008; 46:30213029. [PubMed: 18691605]

69. Libkuman TM, Otani H, Kern R, Viger SG, Novak N. Multidimensional normative ratings for the International Affective Picture System. Behav Res Methods. 2007; 39:326-334. [PubMed: 17695361]

70. Lang PJ, Bradley MM, Cuthbert BN. Emotion, motivation, and anxiety: brain mechanisms and psychophysiology. Biol Psychiatry. 1998; 44:1248-1263. [PubMed: 9861468]

71. Gruhn D, Scheibe S. Age-related differences in valence and arousal ratings of pictures from the International Affective Picture System (IAPS): do ratings become more extreme with age? Behav Res Methods. 2008; 40:512-521. [PubMed: 18522062]

72. Porto WG, Bertolucci PH, Bueno OF. The paradox of age: an analysis of responses by aging Brazilians to International Affective Picture System (IAPS). Rev Bras Psiquiatr. 2011; 33:10-15. [PubMed: 20585743] 
73. Keil A, Freund AM. Changes in the sensitivity to appetitive and aversive arousal across adulthood. Psychol Aging. 2009; 24:668-680. [PubMed: 19739923]

74. Mikels JA, Fredrickson BL, Larkin GR, Lindberg CM, Maglio SJ, Reuter-Lorenz PA. Emotional category data on images from the International Affective Picture System. Behav Res Methods. 2005; 37:626-630. [PubMed: 16629294]

75. Dien J. Evaluating two-step PCA of ERP data with Geomin, Infomax, Oblimin, Promax, and Varimax rotations. Psychophysiology. 2010; 47:170-183. [PubMed: 19761521]

76. Dien J. The ERP PCA Toolkit: an open source program for advanced statistical analysis of eventrelated potential data. J Neurosci Methods. 2010; 187:138-145. [PubMed: 20035787]

77. Wells RE, Yeh GY, Kerr CE, Wolkin J, Davis RB, Tan Y, Spaeth R, Wall RB, Walsh J, Kaptchuk TJ, Press D, Phillips RS, Kong J. Meditation's impact on default mode network and hippocampus in mild cognitive impairment: a pilot study. Neurosci Lett. 2013; 556:15-19. [PubMed: 24120430]

78. Quintana Hernandez DJ, Miro Barrachina MT, Ibanez Fernandez I, del Pino AS, Garcia R Jr, Hernandez JR. Effects of a neuropsychology program based on mindfulness on Alzheimer's disease: randomized double-blind clinical study. Rev Esp Geriatr Gerontol. 2014; 49:165-172. [PubMed: 24880524]

79. van Boxtel MP, Speckens AE. Mindfulness, cognitive function and 'successful ageing'. Tijdschr Gerontol Geriatr. 2014; 45:137-143. [PubMed: 24323197]

80. Larouche E, Hudon C, Goulet S. Potential benefits of mindfulness-based interventions in mild cognitive impairment and Alzheimer's disease: an interdisciplinary perspective. Behav Brain Res. 2015; 276:199-212. [PubMed: 24893317]

81. Quintana Hernandez DJ, Miro Barrachina MT, Ibanez Fernandez I, Santana Del Pino A, Rojas Hernandez J, Rodriguez Garcia J, del Quintana Montesdeoca MP. Mindfulness-based stimulation in advanced Alzheimer's disease: A comparative, non-inferiority, clinical pilot study. Rev Esp Geriatr Gerontol. 2015; 50:168-173. [PubMed: 25796322]

82. Pessoa L. How do emotion and motivation direct executive control? Trends Cogn Sci. 2009; 13:160-166. [PubMed: 19285913]

83. Pessoa L. Emergent processes in cognitive-emotional interactions. Dialogues Clin Neurosci. 2010; 12:433-448. [PubMed: 21319489]

84. Pessoa L, Adolphs R. Emotion processing and the amygdala: from a 'low road' to 'many roads' of evaluating biological significance. Nat Rev Neurosci. 2010; 11:773-783. [PubMed: 20959860]

85. Pessoa L. On the relationship between emotion and cognition. Nat Rev Neurosci. 2008; 9:148-158. [PubMed: 18209732]

86. Mather M, Sutherland MR. Arousal-Biased Competition in Perception and Memory. Perspect Psychol Sci. 2011; 6:114-133. [PubMed: 21660127]

87. Nashiro K, Mather M. Effects of emotional arousal on memory binding in normal aging and Alzheimer's disease. Am J Psychol. 2011; 124:301-312. [PubMed: 21977692]

88. Sakaki M, Fryer K, Mather M. Emotion strengthens high-priority memory traces but weakens lowpriority memory traces. Psychol Sci. 2014; 25:387-395. [PubMed: 24311478]

89. Lee TH, Sakaki M, Cheng R, Velasco R, Mather M. Emotional arousal amplifies the effects of biased competition in the brain. Soc Cogn Affect Neurosci. 2014; 9:2067-2077. [PubMed: 24532703]

90. Mather M, Mitchell KJ, Raye CL, Novak DL, Greene EJ, Johnson MK. Emotional arousal can impair feature binding in working memory. J Cogn Neurosci. 2006; 18:614-625. [PubMed: 16768364]

91. Gavazzeni J, Wiens S, Fischer H. Age effects to negative arousal differ for self-report and electrodermal activity. Psychophysiology. 2008; 45:148-151. [PubMed: 17850240]

92. McCarthy G, Donchin E. A metric for thought: a comparison of P300 latency and reaction time. Science. 1981; 211:77-80. [PubMed: 7444452]

93. Kutas M, McCarthy G, Donchin E. Augmenting mental chronometry: the P300 as a measure of stimulus evaluation time. Science. 1977; 197:792-795. [PubMed: 887923]

94. Zhang Q, Lawson A, Guo C, Jiang Y. Electrophysiological correlates of visual affective priming. Brain Res Bull. 2006; 71:316-323. [PubMed: 17113962] 
95. Koelsch S, Gunter TC, Wittfoth M, Sammler D. Interaction between syntax processing in language and in music: an ERP Study. J Cogn Neurosci. 2005; 17:1565-1577. [PubMed: 16269097]

96. Featherstone CR, Morrison CM, Waterman MG, MacGregor LJ. Semantics, syntax or neither? A case for resolution in the interpretation of $\mathrm{N} 500$ and $\mathrm{P} 600$ responses to harmonic incongruities. PLoS One. 2013; 8:e76600. [PubMed: 24223704]

97. James CE, Cereghetti DM, Roullet Tribes E, Oechslin MS. Electrophysiological evidence for a specific neural correlate of musical violation expectation in primary-school children. Neuroimage. 2015; 104:386-397. [PubMed: 25278251]

98. Luo Y, Shen W, Zhang Y, Feng TY, Huang H, Li H. Core disgust and moral disgust are related to distinct spatiotemporal patterns of neural processing: an event-related potential study. Biol Psychol. 2013; 94:242-248. [PubMed: 23816951]

99. Chi S, Wang C, Jiang T, Zhu XC, Yu JT, Tan L. The prevalence of depression in Alzheimer's disease: a systematic review and meta-analysis. Curr Alzheimer Res. 2015; 12:189-198. [PubMed: 25654505]

100. Drijgers RL, Verhey FR, Leentjens AF, Kohler S, Aalten P. Neuropsychological correlates of apathy in mild cognitive impairment and Alzheimer's disease: the role of executive functioning. Int Psychogeriatr. 2011; 23:1327-1333. [PubMed: 21729413]

101. Korczyn AD, Halperin I. Depression and dementia. J Neurol Sci. 2009; 283:139-142. [PubMed: 19345960]

102. Kumar A, Singh A, Ekavali. A review on Alzheimer's disease pathophysiology and its management: an update. Pharmacol Rep. 2015; 67:195-203. [PubMed: 25712639]

103. Guillem F, Chouinard S, Poulin J, Godbout R, Lalonde P, Melun P, Bentaleb LA, Stip E. Are cholinergic enhancers beneficial for memory in schizophrenia? An event-related potentials (ERPs) study of rivastigmine add-on therapy in a crossover trial. Prog Neuropsychopharmacol Biol Psychiatry. 2006; 30:934-945. [PubMed: 16580765]

104. Reeves RR, Struve FA, Patrick G. The effects of donepezil on quantitative EEG in patients with Alzheimer's disease. Clin Electroencephalogr. 2002; 33:93-96. [PubMed: 12025738] 

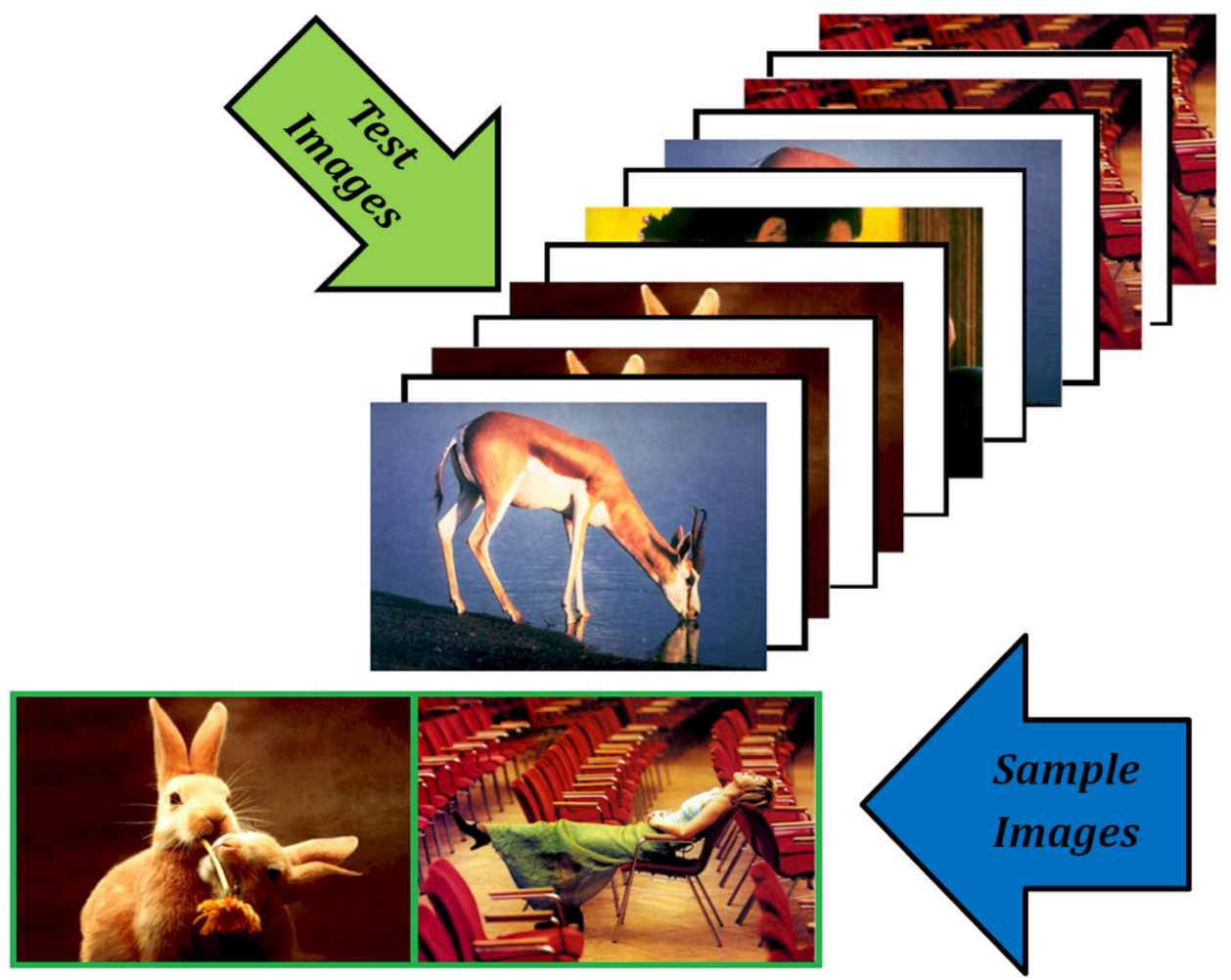

Figure 1. Experimental Schematic

This figure summarizes a typical trial in the current experiment. The z-axis represents the passage of time during the trial. First, two sample images are displayed with a green border, and participants are directed to commit these images to memory. Then, text images are displayed one-by-one, and participants indicate whether each image was among the sample images from that trial by keyboard press. 


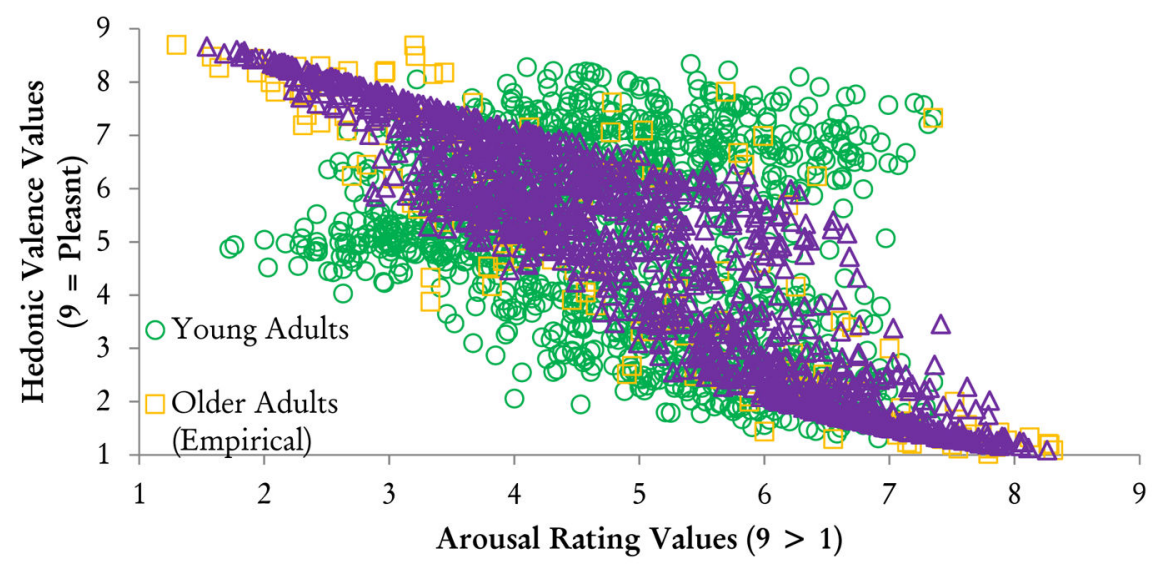

Figure 2. Scatterplot of distribution of hedonic valence and arousal scores of IAPS images This graph depicts younger and older adults' ratings of IAPS images hedonic valence and arousal. In younger adults (green circles), these dimensions are relatively uncorrelated, but the correlation between these dimensions is stronger in older adults' reported scores (orange squares). The results of applying a polynomial ordinary least squares regression algorithm to generate estimated older adult hedonic valence and arousal ratings for all IAPS images (purple triangles) further clarifies this dependent relationship. This dependence led us to encode the emotional enhancement effect levels of the experimental stimuli unidimensionally. 


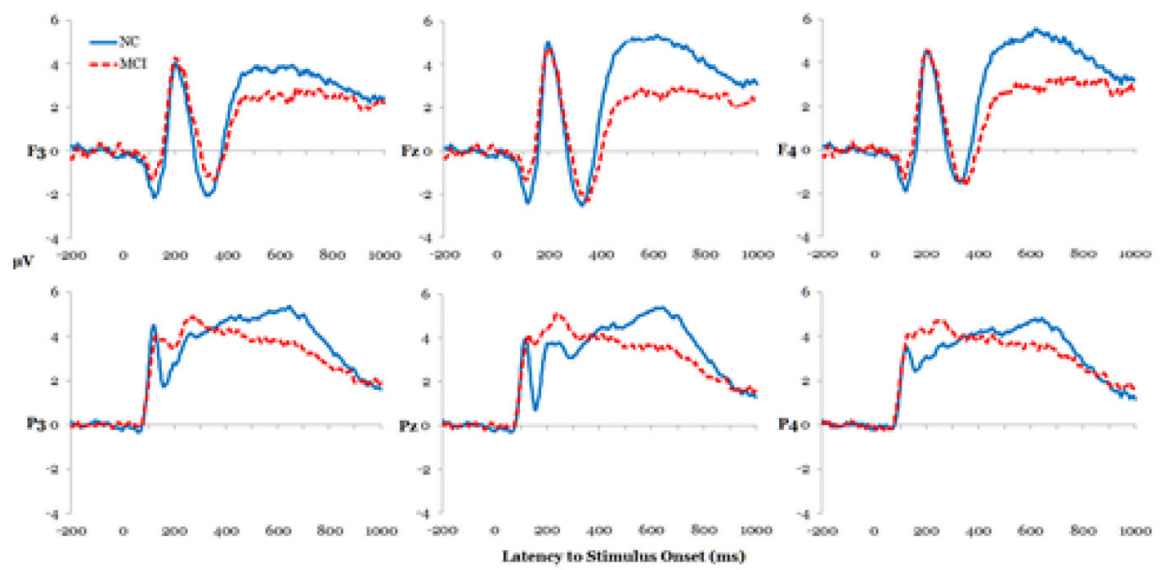

Figure 3. Conventional ERPs separated by clinical group

The grand average waveforms of all experimental conditions and participants by group have been displayed at 6 electrodes. The experiment was confirmed to have evoked conventional emotional working memory ERP/EEG waveforms, permitting further analysis. 

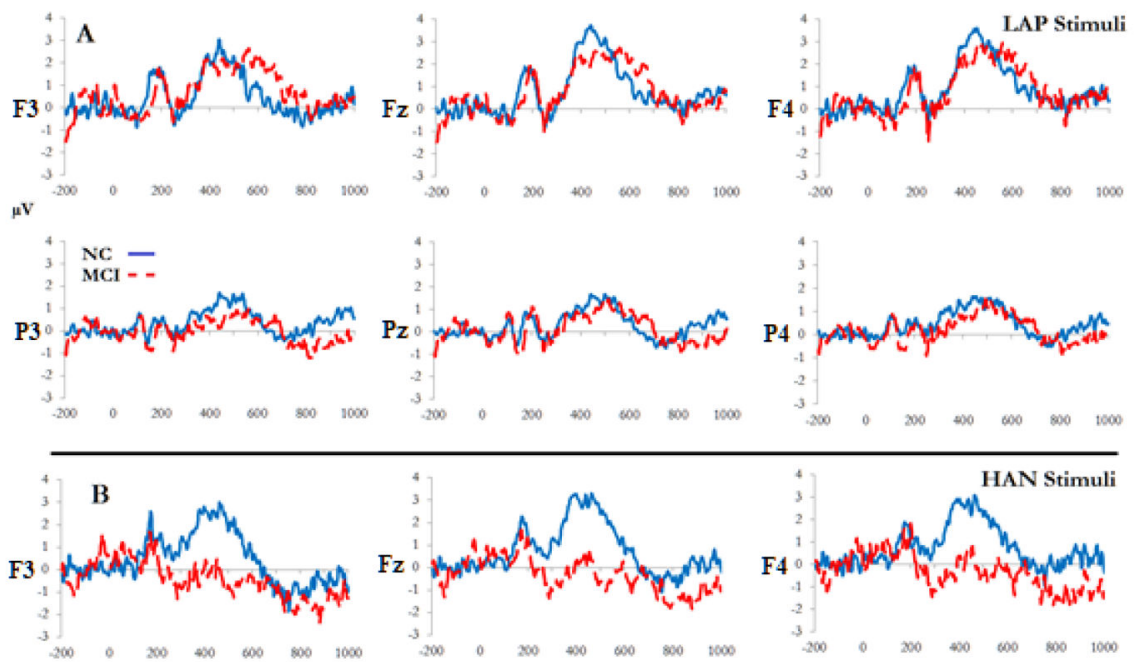

$\mu \mathrm{V}$

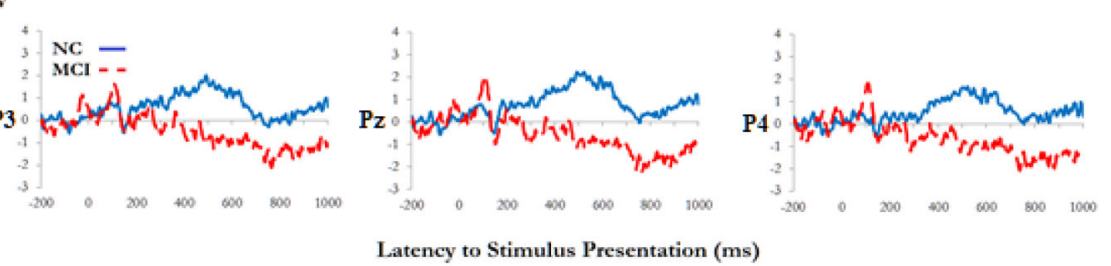

Figure 4. Working memory difference waves for LAP and HAN stimuli

Averaged ERPs for persons without impairment (NC) and persons with MCI (MCI) for low arousal positive stimuli (A) and high arousal negative stimuli (B). Differences between groups appeared most obvious for HAN stimuli in the later time-window; however, because the results of the temporal PCA suggested overlapping individual components in this window, statistical analysis was deferred to the related temporal components. 

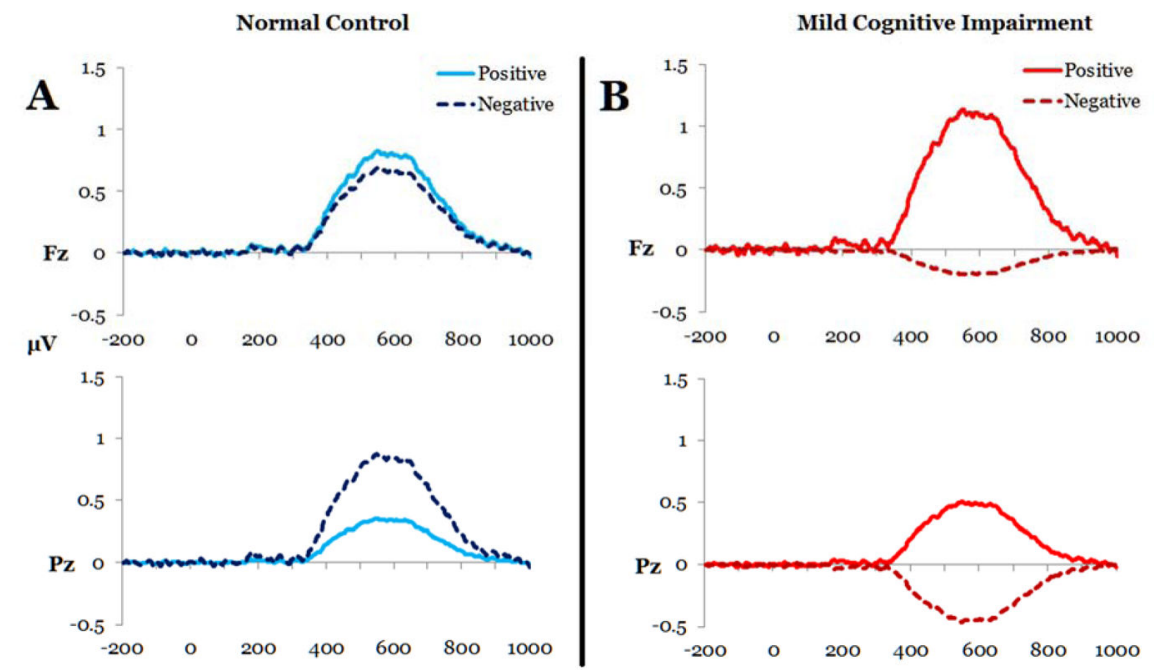

Figure 5. Summary of the group differences at the $\mathrm{P600}$ as difference waves Each line depicts the difference in P600 activity between matching and non-matching stimuli for stimuli that were either positive (solid line) or negative (dashed line). Individuals without impairment (A) and individuals with MCI (B) showed similar brain responses for positive stimuli, but very different responses for negative stimuli. Data have been graphed at a frontal $(\mathrm{Fz})$ and posterior $(\mathrm{Pz})$ electrode in the first and second rows, respectively, to provide a general sense of differences in this effect at frontal and posterior sites. 


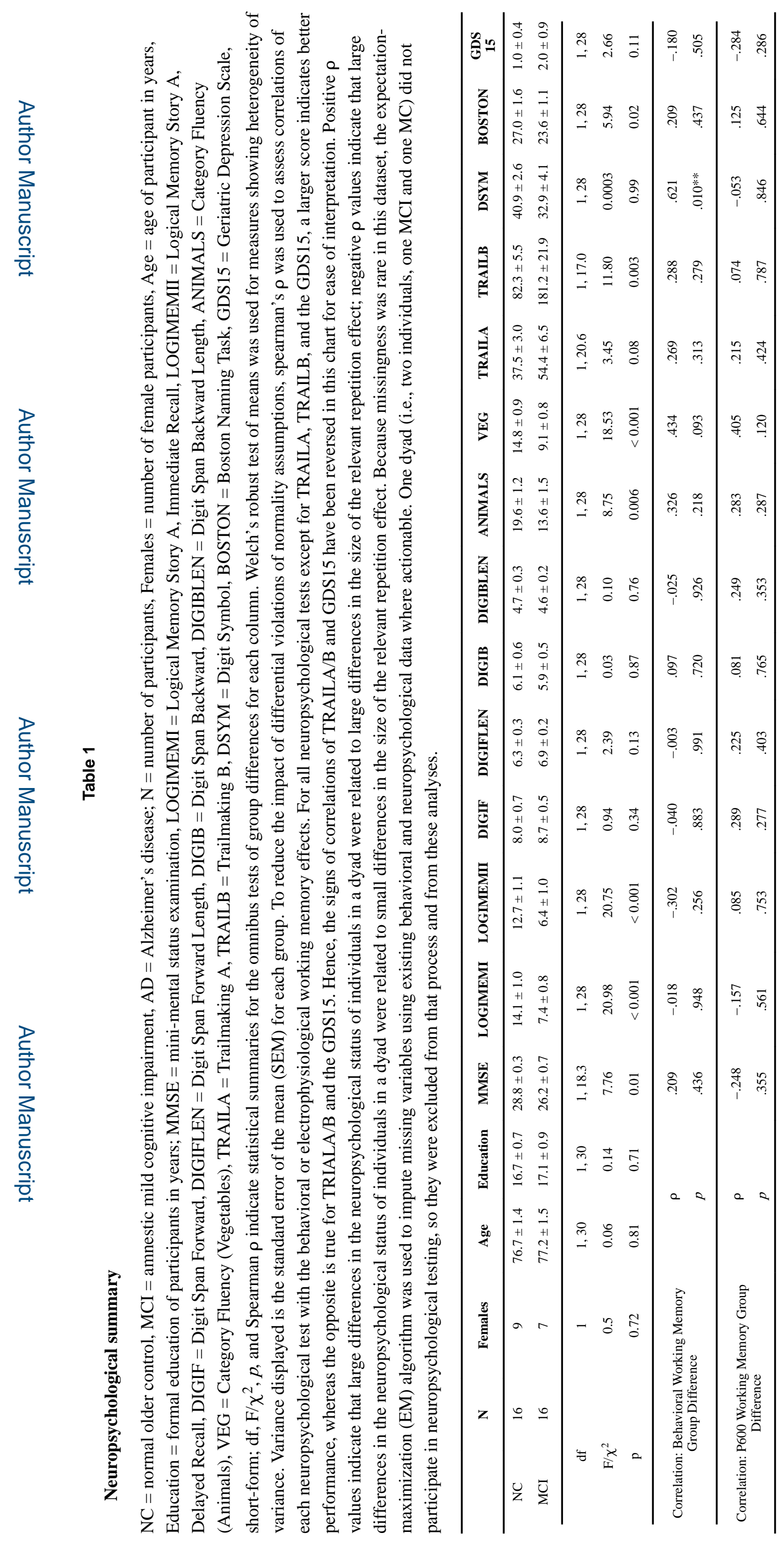




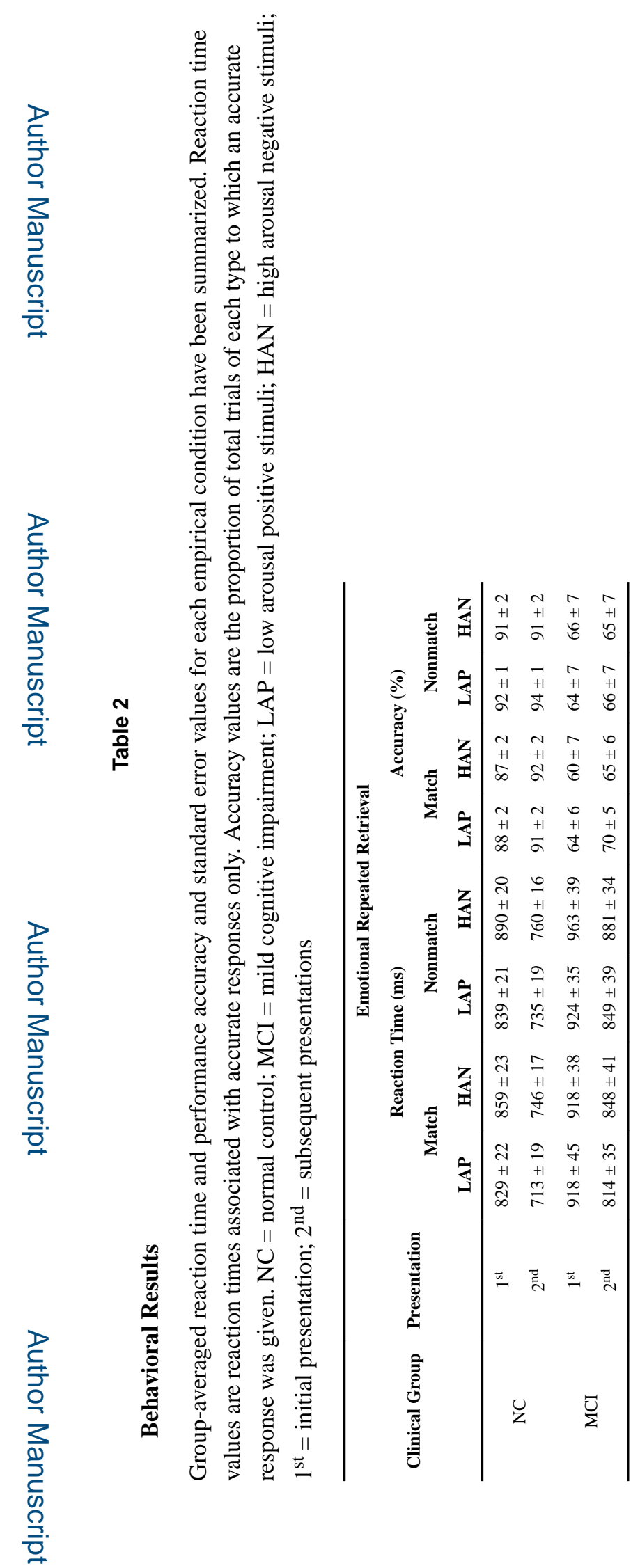

J Alzheimers Dis. Author manuscript; available in PMC 2018 May 01. 